\title{
Hip development after surgery to prevent hip dislocation in cerebral palsy: a longitudinal register study of 252 children
}

\author{
Philippe WAGNER ${ }^{1,3}$ and Gunnar HÄGGLUND ${ }^{1,2}$
}

\author{
${ }^{1}$ Lund University, Department of Clinical Sciences, Lund, Orthopedics; ${ }^{2}$ Department of Orthopedics, Skane University Hospital, Lund; ${ }^{3}$ Centre for Clinical \\ Research, Uppsala University, Region Västmanland, Västerås, Sweden \\ Correspondence: philippe.wagner@regionvastmanland.se \\ Submitted 2021-06-25. Accepted 2021-09-14.
}

Background and purpose - Operative treatment of hip displacement in cerebral palsy $(\mathrm{CP})$ includes adductor-psoas lengthening (APL) or varus derotation osteotomy (VDRO) of the proximal femur, sometimes combined with pelvic osteotomy. After both operations, there is a significant risk of relapse and need for reoperation. We used the migration percentage (MP) to compare the development of hip displacement after APL and VDRO.

Patients and methods - All reported MP measurements for children treated with APL $(\mathrm{n}=158)$ or VDRO ( $\mathrm{n}$ $=94$ ) and followed $\geq 3$ years were obtained from the Swedish Surveillance Programme for CP. In children treated with bilateral surgery, the hip with the highest preoperative MP was analyzed. A mixed-effects model was used to estimate the development of MP with age for each child and the population mean.

Results - The 104 hips that underwent APL without reoperation showed a gradually reduced MP postoperatively. The 54 hips that underwent a 2nd surgery with APL or VDRO because of redisplacement had a higher displacement rate preoperatively and continued displacement postoperatively but at a slower rate. The 94 hips that underwent VDRO showed an instantaneous decrease in MP postoperatively followed by a rate of increasing displacement. The 15 hips that underwent a 2nd VDRO had a higher rate of displacement both pre- and postoperatively.

Interpretation - After APL, hip displacement either decreases or continues to increase but at a slower rate. After VDRO, the MP decreases momentarily but then gradually increases. The risk of reoperation is higher in children with a high preoperative displacement velocity and a high preoperative MP.
Hip dislocation occurs in 15-20\% of children with cerebral palsy (CP) if not prevented (1). The risk is highest in children with severe limitations of gross motor function and occurs predominantly in those with the spastic or dyskinetic subtypes $(2,3)$. In most cases, hip dislocation is preventable by followup with repeated radiographic examinations $(4,5)$.

The operative treatment of hip displacement in $\mathrm{CP}$ includes adductor-psoas lengthening (APL) or varus derotation osteotomy (VDRO) of the proximal femur, sometimes in combination with pelvic osteotomy. There are different opinions about when and the type of surgery needed, especially in relation to the child's age, degree of displacement, and gross motor function level. Regardless of the surgical procedure, there is a high risk that repeat surgery is needed during the child's remaining growth period (6-10).

The Swedish Cerebral Palsy Follow-up Program (CPUP) started in southern Sweden (1.2 million inhabitants) in 1994 and since 2006 has included $>95 \%$ of all children and adolescents with CP in Sweden born in 2000 and later (11). In CPUP, the hips are followed with repeated measurements of the migration percentage (MP) on anteroposterior (AP) pelvic radiographs (12).

We used data from CPUP to analyze the development of hip displacement as measured with the MP after APL and VDRO and compared hips with and without a 2nd operation.

\section{Patients and methods}

In CPUP, the CP diagnosis is determined by a neuropediatrician. The exclusion and inclusion criteria are in accordance with the Surveillance of Cerebral Palsy Network in Europe 
(13). Gross motor function is classified by the child's physiotherapist according to the Gross Motor Function Classification System (GMFCS), which is an age-related system with 5 levels ranging from $\mathrm{I}$ to $\mathrm{V}$, in which children with level $\mathrm{V}$ are affected the most (14).

Children in GMFCS levels III-V are examined radiographically with a pelvic AP view once a year, and those in level II at 2 and 6 years of age. Children in level I are not examined radiographically if they have a normal pain-free range of movement of the hip. After age 8 years, children are followed individually according to the results of their previous clinical and radiological findings.

In Sweden, surgical procedures to prevent hip dislocation in children with CP are performed in 7 university hospitals and 5 regional hospitals (10). Preventive surgery is recommended when the MP is $>40 \%$. However, there are no specific recommendations regarding when APL or VDRO should be performed, or when a VDRO should be combined with pelvic osteotomy.

In this study, the reported preoperative and all postoperative radiographs for all children in GMFCS levels III-V born in 2000-2011 treated with APL or VDRO before the age of 12 years were analyzed. Each child was included in the APL or VDRO group according to their 1st operation. In each group, the hip with the highest MP preoperatively was included. To obtain usable longitudinal data to describe the individual child MP development, only children followed for at least 3 years before the age of 12 years were included and their measurements only up to age 12 years. Children who received a reoperation because of redisplacement within the study period were analyzed separately. Data for each child from the 1st clinical visit to the last, 2nd operation, or December 31, 2017 were included in the analyses.

\section{Statistics}

The statistical analysis focused on the development of MP from 5 years before to 5 years after the operation. Because population trends may not necessarily represent individual developments, a mixed-effects model (MM) was used to estimate the variation in individual development of MP over time as well as the population trend. The MM has the advantage of accounting for the correlation structure imposed by repeated measurements on the same leg and of adjusting for potential bias because of dropouts (15).

The MM included a 2nd-degree polynomial of time from surgery with random effects for each coefficient, which allowed us to generate separate 2nd-degree polynomials to predict the mean MP development for each individual child. We used an interaction term to identify different 2 nd-degree polynomials before and after the operation, both for the population mean, in terms of fixed effects, and for individual children in terms of random effects. The Akaike information criterion was then used to select the most parsimonious MM possible with respect to both fixed and random effects. Here, the results of the full model are compared with those of all nested models for both fixed and random effects. The fit of the final model was ensured using residual plots. The analysis was stratified into the APL and VDRO groups, and according to receipt of a 2nd operation. The results are presented using means, mean differences, standard deviations (SD), and corresponding $95 \%$ confidence intervals (CIs) together with graphs of predicted MP development with time before and after operation for each stratum. The data are presented as the population mean with $\mathrm{CI}$, together with an interval including all individual means within $1 \mathrm{SD}$ of the population mean.

$\mathrm{MM}$ analysis was also used to analyze differences between those having had a 2nd operation and those who had not, stratified by operation type, APL or VDRO. The MM was of the same form as previous models with the addition of an indicator variable for having had a 2 nd operation. Further analyses were performed to identify differences between children receiving a VDRO with and without pelvic osteotomy by including in the final MM used to describe the VDRO cohort an interaction term between a pelvic osteotomy indicator variable and all time-related variables. Model selection was then performed as previously described.

To examine additional factors that may differ between those with and without a 2 nd operation, we performed additional analyses: linear regression to test differences in mean age at operation and proportional odds regression to examine differences in GMFCS distribution. In both models examined variables were used as outcome and an indicator variable for those with a 2nd operation was used as explanatory variable.

Because children are not generally examined within 6 months after surgery, we excluded this time interval from the analysis. This is because any examination performed during this time would likely reflect the need for close monitoring and, consequently, such measurements may not be representative of the cohort of primary interest.

\section{Ethics, funding, and potential conflicts of interest}

The study was approved by the Medical Research Ethics Committee at Lund University (LU-443-99), and permission was obtained to extract data from the CPUP registry. The study was funded by Stiftelsen för bistånd åt rörelsehindrade i Skåne. The authors declare no conflict of interest.

\section{Results}

The study was based on 1,589 radiographs from 252 children: 158 children (96 boys) were treated with APL at age 5.3 years (1.5-12) and 94 children (54 boys) with VDRO at age 6.7 years (2.1-12.0). VDRO was combined with a pelvic osteotomy in 43 of the hips (Table).

In the 104 hips that underwent APL without a 2nd operation during the follow-up period the MM that best fit the data was one with a linear trend with time both before and after 
Number of children according to GMFCS level, sex, and performed preventive surgery. Data are number of children, with the number reoperated in parentheses

\begin{tabular}{lrllllr}
\hline & \multicolumn{4}{c}{ GMFCS level } & \multicolumn{2}{c}{ Sex } \\
Factor & III & IV & V & Boys & Girls & Total \\
\hline APL & $16(4)$ & $57(15)$ & $85(35)$ & $96(31)$ & $62(23)$ & $158(54)$ \\
VDRO, total & $5(0)$ & $31(5)$ & $58(10)$ & $54(10)$ & $40(5)$ & $94(15)$ \\
$\quad \quad$ pelvic osteotomy & $1(0)$ & $16(1)$ & $26(2)$ & $21(3)$ & $22(0)$ & $43(3)$ \\
Total & $21(4)$ & $88(21)$ & $143(45)$ & $150(41)$ & $102(28)$ & $252(69)$ \\
\hline
\end{tabular}

All reoperations after primary VDRO consisted of new VDRO.

Reoperations after primary APL consisted of VDRO in 51 children and new APL in 3 children.

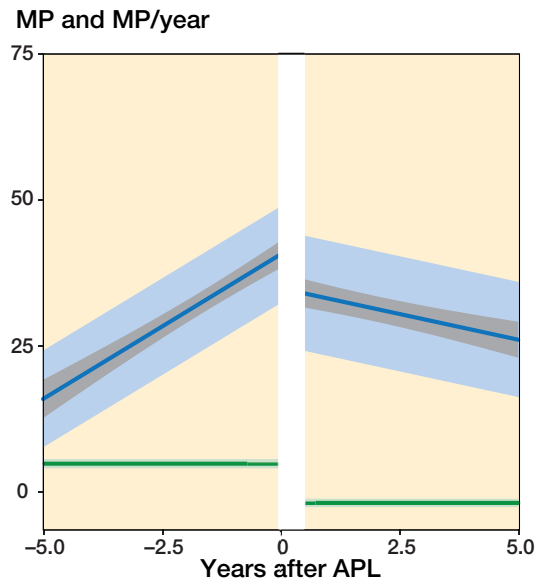

Figure 1. Predicted mean MP development with time before and after APL without a 2nd operation at the end of follow-up. Population mean with $95 \% \mathrm{Cls}$ (gray), and 1 SD of the individual means (light blue). Predicted mean MP velocity (MP/year) and the $95 \% \mathrm{Cl}$ (green).

MP and MP/year

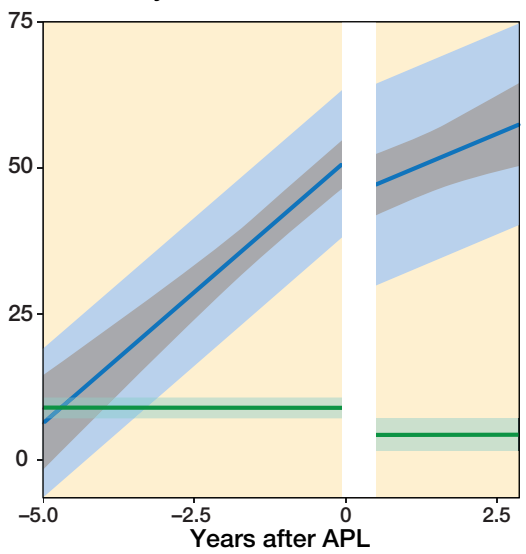

Figure 2. Predicted mean MP development and MP velocity with time before and after the APL with a 2nd operation at the end of follow-up. The indicators are as described for Figure 1.

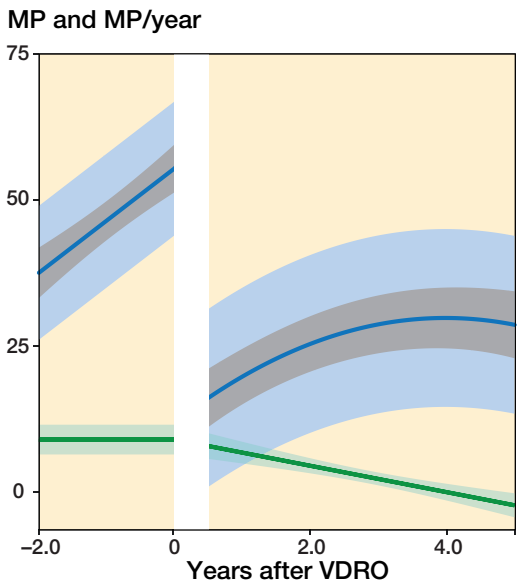

Figure 3. Predicted mean MP development for patients who underwent a VDRO without a 2nd operation at the end of follow-up. The indicators are as described for Figure 1

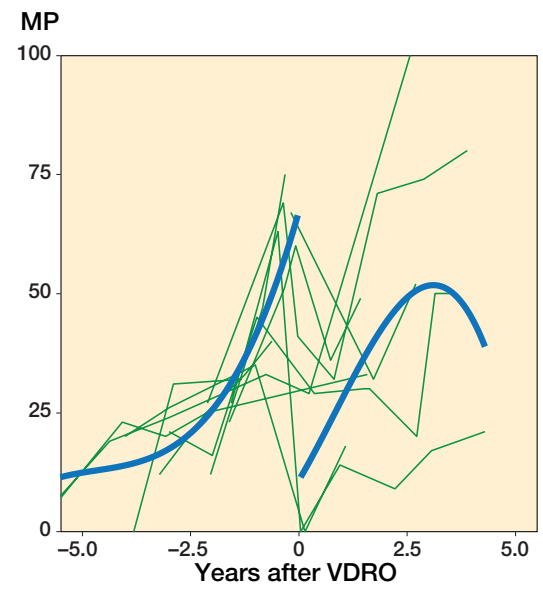

Figure 4. Individual MP measurements and the population mean plotted against time before and after the operation for patients who underwent a VDRO with a 2nd operation at the end of the follow-up. Patients were followed until the time of their 2nd operation. The population mean was estimated using a cubic spline. the operation together with a random intercept to describe the individual mean developments. These hips had a gradually reduced MP during the 5 years postoperatively (Figure 1). The displacement velocity was $4.9 \% / y e a r(\mathrm{CI} 4.2-5.7)$ preoperatively and the reduction velocity was $1.8 \%$ /year (CI 1.1-2.5) postoperatively. The mean age at the time of the operation was 5.7 years (1.9-12) and the mean MP (estimated from the model) was 41\% (CI 39-43), SD 8.3 (CI 6.8-10).

In the 54 hips that underwent APL and a 2nd operation the MM that best fit the data was also a linear trend with time both before and after the operation together with a random intercept to describe the individual mean developments. Because the data was sparse beyond 2.5 years after APL (Figures 5-6, see Supplementary data), all remaining analyses were performed using data until 2.5 years after surgery only. The hips showed a displacement velocity of 9.0\%/year (CI 7.2-11) preoperatively followed by a further but reduced velocity of $4.4 \% /$ year (CI 1.6-7.1) postoperatively (Figure 2). The mean age at the time of the primary operation was 4.6 years (range $1.5-10$ ), and the mean MP (estimated from the model) was 51\% (CI 47-56), SD 13 (CI 10-16). Analysis of the pooled data produced a significant difference $(\mathrm{p}<0.001)$ in displacement velocity before surgery between hips that underwent a 2nd operation and those that did not. Children with a 2 nd operation were younger, on average 1.1 years (CI 0.3-1.9) and had increased odds of having a higher GMFCS level, OR 1.2 (CI 1.0-9.4).

In the 79 hips that underwent VDRO without a 2nd operation during the follow-up period the MM that best fit the data 
was also a linear trend with time before the operation and a 2nd-degree polynomial trend with time after the operation together with a random intercept to describe the individual mean developments. The hips showed an instantaneous reduction of MP postoperatively from 55\% (CI 51-60), SD 13 (10-16) to $11 \%$ (CI 6.1-17), SD 15.6, which represents a mean reduction of $44 \%$ (CI 37-51), SD 9.9 (CI 6.2-16). This was followed by a recurring displacement at a gradually decreasing velocity from 6.8\%/year (CI 5.0-8.7) 1 year postoperatively to $0 \% /$ year (CI $-1.5-1.4$ ) after 4 years (Figure 3 ). Preoperatively, the displacement velocity was $9.1 \%$ year (CI 6.6-12). The mean age at the time of the operation was 7.0 years (range 2.1-12).

The 15 hips that underwent VDRO and a 2nd operation appeared to have a higher displacement velocity both preoperatively and postoperatively than those that were not reoperated (Figure 4). However, due to the limited number of patients, we performed no formal analysis on this group alone. Their mean age at the time of the primary operation was 5.2 years (3.2-9.9).

Because data for hips that underwent a 2nd operation was sparse and the time trend patterns complex, as shown in Figure 4 and Figure 7 (see Supplementary data), we performed pooled analyses using the data for up to 2.5 years after surgery only. The differences between hips that did and did not undergo a 2nd operation after VDRO were statistically significant. Hips that underwent a 2nd operation had a higher displacement velocity before surgery, with a difference of $10 \%$ /year (CI 2.6-17). At the time of surgery, the MP was slightly higher (3.9\%, CI -6.4 to 14$)$ and the displacement velocity was higher (6.8\%/year, CI -6.1 to 20) after the operation. Children with a 2nd operation were younger on average, 1.8 years (CI 0.5-3.2) and had increased odds of having a higher GMFCS level, OR 1.4 (CI 0.5-4.8).

Hips that underwent a pelvic osteotomy had a higher MP at the time of the operation (3.3\%, CI -2.6 to 9) (Figure 8, see Supplementary data). After the operation, hips that underwent a VDRO with a pelvic osteotomy had a generally lower MP than those treated with a VDRO only $(18 \%$, CI 10-26).

\section{Discussion}

Our main findings were that children who underwent APL showed a decreasing displacement or a reduced displacement rate postoperatively, whereas those who underwent a VDRO had a momentary decrease in MP but after that developed gradually increasing hip displacement. Children who underwent a 2 nd operation had a higher rate of displacement and higher MP preoperatively.

Our choice of statistical methods has both limitations and strengths. In addition to allowing for adjustment for bias because of dropouts, our methods allowed us to describe the mean trends at both the individual patient and popula- tion levels. This added important information to the analysis by allowing us to evaluate how well the population mean described the mean development for each child. A large variation in individual means would indicate heterogeneous developmental trends and that the population mean did not describe well the mean trend for each child. On the other hand, a small variation would indicate a more homogeneous development pattern among the study participants and that the population mean described well this common pattern. This aspect may be particularly important when considering the change in MP after surgery because studying only the population mean may hide differences in results between children.

Another strength of our approach relates to the potential for bias because of possible regression to the mean (16). Commonly, regression to the mean effects is adjusted for by conditioning the analyses on baseline outcome measurements. In the current analysis, this was done partly by including a random intercept for each patient, which may have been beneficial in compensating for these effects.

A possible limitation of our study is that the distribution and measurements for each child may not have been sufficient to describe the mean individual development accurately. This may explain why a model including a simple random intercept for each child, as opposed to a more complex random coefficients model, appeared to be the best fitting statistical model. Future studies involving more measurements and more extensive follow-up for each child could reveal a more complex developmental pattern. This should be especially true for the model we used to describe the differences between hips undergoing a VDRO with and without pelvic osteotomy, where the parallel pattern, as shown in Figure 8 (see Supplementary data), could be an oversimplification; therefore, this graph should be interpreted with caution.

An additional limitation is that we did not apply a stricter criterion for the years of follow-up needed for study inclusion. With the current criteria, some children may not have follow-up of sufficient duration to have had a 2 nd operation registered in the study data. It is possible that misclassification may have occurred and biased the results by underestimating differences between those having had a 2 nd operation and those who had not.

In a prospective follow-up of 31 children who underwent APL, Terjesen (8) found a higher preoperative migration rate (11\%/year) in children with a poor outcome than in those with a good outcome (5\%/year), although the difference was not statistically significant. In that study, a preoperative MP $\geq$ $50 \%$ was a risk factor for poor outcome. In a retrospective review of 330 children who underwent APL, Shore et al. (6) also found that a high preoperative MP was associated with a poorer outcome and that the failure rate increased with higher GMFCS level. However, a multivariable analysis that adjusted for preoperative MP found that children in GMFCS levels III-V had a similar failure risk, as also found in our study and in Terjesen (8). 
In a retrospective analysis of 320 children who underwent a VDRO, Shore et al. (7) found that young age at surgery was associated with the need for later revision surgery and that soft tissue release during the VDRO decreased the need for revision. The revision rate was higher for children in GMFCS level IV or V than in those in level I or II. In a prospective study of 31 children who underwent a VDRO, Terjesen (9) found a higher risk of a poor outcome in children in GMFCS level $\mathrm{V}$ than in those in level IV and that a high MP 1 year after the operation was a risk factor for the need for later revision surgery.

Hip dislocation in $\mathrm{CP}$ is caused by altered muscle forces on the hip joint. The stronger and more spastic adductor and flexor muscles cause hip positioning in adduction, flexion, and inward rotation, which shifts the force vector from the anteromedial to posterolateral direction (17). This suggests that the mechanism responsible for the reduced risk of hip displacement after APL creates conditions for positioning the hip in a more favorable position. After the varization during the VDRO, the hip abduction may be initially restricted with positioning in greater adduction, which produces a less favorable force direction. This may explain the increasing risk of displacement after VDRO. We have found that the passive range of abduction increased by an average of $10^{\circ}$ during the 18-36 months after APL but remained unchanged after VDRO (Hägglund and Wagner 2021, personal communication). This may also explain the finding of Shore et al. (7) that soft tissue release during VDRO protects against the need for revision surgery. However, we do not have registry data that allows us to analyze the postoperative development in relation to the degree of varization, the degree of shortening, and concomitant soft tissue surgery.

Children who underwent a 2nd operation had a faster increase in MP before the primary surgery, higher MP preoperatively, and were operated on at a younger age. These findings are interrelated and may all be the consequence of a greater muscle imbalance on the hip joint in these children.

The ideal management is to prevent hip dislocation with as limited surgery as possible performed before joint deformities develop and to avoid the need for repeated surgery. Based on the results of this study, it seems appropriate to perform an early APL on hips at risk for dislocation. Young age at first examination in the follow-up program, high GMFCS level, and a high head-shaft angle are known risk factors for hip displacement. Based on these factors, the CPUP Hip Score was developed and has been shown to have a good ability to differentiate between high- and low-risk individuals (18).

In conclusion, after APL, hip displacement either decreases or continues to increase but at a slower rate. By contrast, after VDRO, the MP decreases momentarily in most patients but then gradually increases. The risk of reoperation is higher in children with a high preoperative displacement rate and a high preoperative MP. These findings support the importance of early operative treatment of children at risk for hip dislocation.
Study design: GH, PW. Data collection: GH, PW. Statistical analysis: PW. Manuscript preparation: GH, PW.

Acta thanks Niels Wisbech Pedersen and other anonymous reviewers for help with peer review of this study.

1. Hägglund G, Andersson S, Düppe H, Lauge-Pedersen H, Nordmark E, Westbom L. Prevention of hip dislocation in children with cerebral palsy: the first ten years' experience of a population-based prevention programme. J Bone Joint Surg 2005; 87-B: 95-101.

2. Soo B, Howard J J, Boyd R N, Reid S M, Lanigan A, Wolfe R, Reddihough D, Graham H K. Hip displacement in cerebral palsy. J Bone Joint Surg Am 2006; 88: 121-9.

3. Hägglund G, Lauge-Pedersen H, Wagner P. Characteristics of children with hip displacement in cerebral palsy. BMC Musculoskel Disord 2007; 8: 101.

4. Hägglund G, Alriksson Schmidt A, Lauge Pedersen H, Rodby Bousquet $\mathbf{E}$, Westbom L. Prevention of hip dislocation in children with cerebral palsy: twenty years' result of a population-based prevention programme. Bone Joint J 2014; 96-B: 1546-52.

5. Wordie S J, Robb J E, Hägglund G, Bugler K E, Gaston M S. Hip displacement and dislocation in a total population of children with cerebral palsy in Scotland: status after five years' hip surveillance. Bone Joint $\mathrm{J}$ 2020; 102-B: 383-7.

6. Shore B J, Yu X, Desai S, Selber P, Wolfe R, Graham H K. Adductor surgery to prevent hip displacement in children with cerebral palsy: the predictive role of the Gross Motor Function Classification System. J Bone Joint Surg Am 2012; 94: 326-34.

7. Shore B J, Zurakowski D, Dufreny C, Powell D, Matheney T H, Snyder BD. Proximal femoral varus derotation osteotomy in children with cerebral palsy: the effect of age, Gross Motor Function Classification System level, and surgeon volume on surgical success. J Bone Joint Surg Am 2015; 97: 2024-31.

8. Terjesen T. To what extent can soft-tissue releases improve hip displacement in cerebral palsy? Acta Orthop 2017; 88: 695-700.

9. Terjesen T. Femoral and pelvic osteotomies for severe hip displacement in nonambulatory children with cerebral palsy: a prospective population-based study of 31 patients with 7 years' follow-up. Acta Orthop 2019; 90: 614-21.

10. Kiapekos N, Broström E, Hägglund G, Åstrand P. Primary surgery to prevent hip dislocation in children with cerebral palsy in Sweden: a 5-year follow-up by the national surveillance program (CPUP). Acta Orthop 2019; 90: 495-500.

11. Alriksson-Schmidt A I, Arner M, Westbom L, Krumlinde-Sundholm L, Nordmark E, Rodby-Bousquet E, Hägglund G. A combined surveillance program and quality register improves management of childhood disability. Disabil Rehabil 2017; 39: 830-6.

12. Reimers J. The stability of the hip in children: a radiological study of the results of muscle surgery in cerebral palsy. Acta Orthop Scand 1980; 184(Suppl.): 1-100.

13. Surveillance of Cerebral Palsy Network in Europe (SCPE). Surveillance of cerebral palsy in Europe: a collaboration of cerebral palsy surveys and registers. Dev Med Child Neurol 2000; 42: 816-24.

14. Palisano R, Rosenbaum P, Walter S, Russell D, Wood E, Galuppi B. Development and reliability of a system to classify gross motor function in children with cerebral palsy. Dev Med Child Neurol 1997; 39: 214-23.

15. Mallinckrodt C H, Clark W S, David S R. Accounting for dropout bias using mixed-effects models. J Biopharm Stat 2001; 11: 9-21.

16. Linden A. Assessing regression to the mean effects in health care initiatives. BMC Med Res Methodol 2013; 13: 119.

17. Miller F, Slomczykowski M, Cope R, Lipton G E. Computer modeling of the pathomechanics of spastic hip dislocation in children. J Pediatr Orthop 1999; 19: 486-92.

18. Hermanson M, Hägglund G, Riad J, Rodby-Bousquet E, Wagner P. Prediction of hip displacement in children with cerebral palsy: development of the CPUP Hip Score. Bone Joint J 2015; 97-B: 1441-4. 


\section{Supplementary data}

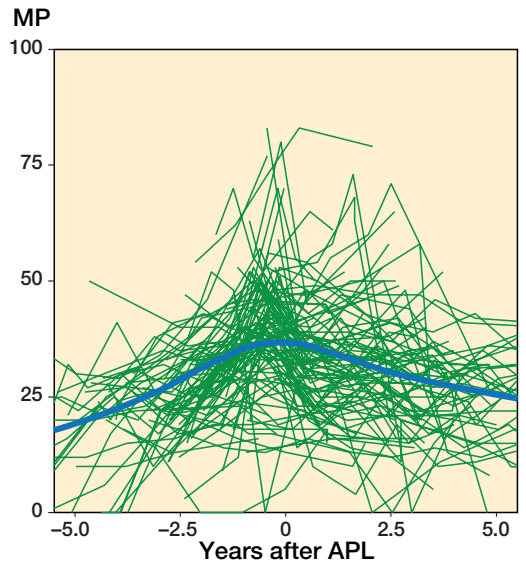

Figure 5. Individual MP measurements and the population mean plotted against time before and after APL for patients who did not undergo a 2nd operation. The population mean was estimated using a local regression (LOESS) smoother.

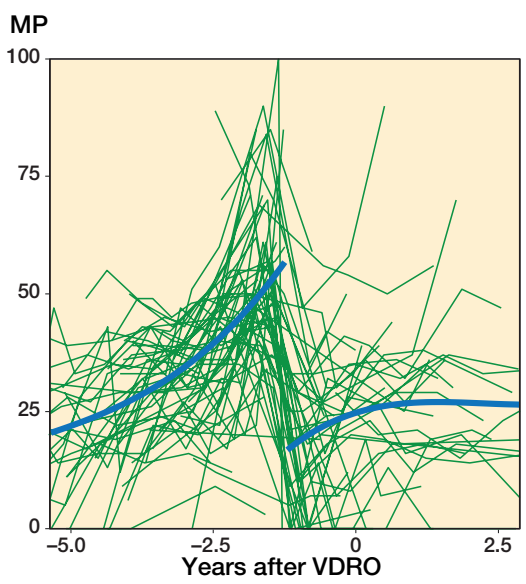

Figure 7. Individual MP measurements and the population mean plotted against time before and after a VDRO for patients who did not undergo a 2nd operation. The population mean was estimated using local regression (LOESS) smoother.

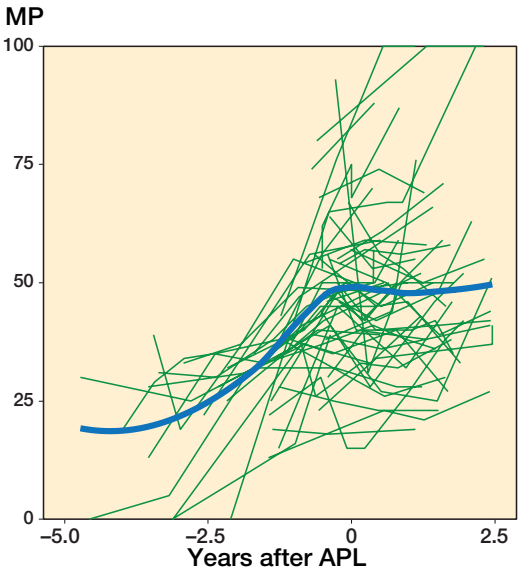

Figure 6. Individual MP measurements and the population mean plotted against time before and after APL. Patients were followed until the time of their 2 nd operation. The population mean was estimated using a local regression (LOESS) smoother.

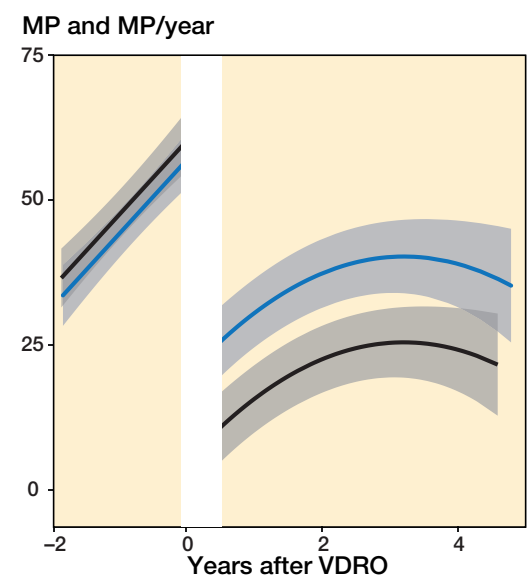

Figure 8. Predicted mean MP development for patients who underwent a VDRO, with and without pelvic osteotomy, and without a 2nd operation at the end of follow-up. The population mean is indicated by the black line for VDRO + pelvic osteotomy and a blue line for VDRO without pelvic osteotomy; the $95 \% \mathrm{Cl}$ is indicated in gray. 